\title{
Yield and Safety of Transjugular Versus Percutaneous Liver Biopsies in Suspected Cases of Diffuse Liver Disease and Correlation of Yield of Transjugular Liver Biopsy with Hepatic Venous Pressure Gradient
}

\author{
${ }^{1}$ Division of Radiodiagnosis, Department of Intervention Radiology, \\ Indian Institute of Liver and Digestive Sciences, Kolkata, \\ West Bengal, India \\ 2Department of Radiodiagnosis, Ruby General Hospital, Kolkata, \\ West Bengal, India \\ ${ }^{3}$ Department of Radiology and Imaging, Tata Medical Center, \\ Kolkata, West Bengal, India \\ ${ }^{4}$ Division of General Medicine, Department of Gastroenterology, \\ IPGMER and SSKM Hospital, Kolkata, West Bengal, India \\ ${ }^{5}$ Department of Pathology, GI Pathology, School of Digestive and \\ Liver Diseases, IPGMER, Kolkata, West Bengal, India \\ 6 Department of Gastroenterology, Indian Institute of Liver and \\ Digestive Sciences, Kolkata, West Bengal, India
}

Mousam Dey ${ }^{1}$ Simi Das ${ }^{2}$ Argha Chatterjee ${ }^{3} \quad$ Agnibha Dutta $^{4} \quad$ Ranajoy Ghosh $^{5} \quad$ Jayanta Dasgupta ${ }^{6}$

\author{
Address for correspondence Mousam Dey, MD, Division of \\ Radiodiagnosis, Department of Vascular Radiology, Indian Institute \\ of Liver and Digestive Sciences, Kolkata, West Bengal, India \\ (e-mail: deymousam@gmail.com).
}

J Gastrointestinal Abdominal Radiol ISGAR 2021;4:3-7.

\section{Abstract \\ Keywords \\ - percutaneous liver biopsy \\ - trans-jugular liver biopsy \\ - hepatic venous pres- sure gradient \\ - complete portal tracts}

Background Liver biopsy is indicated in both diagnosis and prognosis of diffuse liver diseases. Conventionally, percutaneous liver biopsy (PLB) is used, as it is easily available, affordable and has a shorter procedure time, whereas transjugular liver biopsy (TJLB) is used in the setting of ascites and coagulopathy. Our aim is to evaluate the diagnostic yield of TJLB in comparison to PLB with tract embolization. Our secondary aims were to evaluate whether there is any difference in rate of major and minor complications between the two procedures and evaluate whether there is any correlation between diagnostic yield of TJLB and hepatic venous pressure gradient (HVPG).

Methods In this retrospective study, we included a total of consecutive 123 patients who underwent liver biopsy through percutaneous $(n=97)$ and transjugular route $(n=26)$. We compared the yield of the specimen based on the number of complete portal tracts (CPT).

Results There was no significant difference between mean CPT in TJLB and PLB specimens (mean CPT of TJLB and PLB were $10.9 \pm 2.7$ and $11.6 \pm 2.5$, respectively $[p=0.566])$. There was a moderate but significant negative correlation between the total number of CPT and HVPG in the TJLB group (Spearman's rho -0.58$)(p=0.002)$. There was no statistically significant difference in minor complication between the two procedures. Only one patient who underwent PLB developed major complication and none of TLJB procedure had any major complication.

Conclusion Yield of tissue and complication rates are comparable in TJLB and PLB groups. Yield of tissue in TJLB have intermediate but significant negative correlation with HVPG. published online

September 10, 2020
DOI https://doi.org/ 10.1055/s-0040-1716605 ISSN 2581-9933.
(C2020. Indian Society of Gastrointestinal and Abdominal Radiology. This is an open access article published by Thieme under the terms of the Creative Commons Attribution-NonDerivative-NonCommercial-License, permitting copying and reproduction so long as the original work is given appropriate credit. Contents may not be used for commercial purposes, or adapted, remixed, transformed or built upon. (https://creativecommons.org/licenses/by-nc-nd/4.0/).

Thieme Medical and Scientific Publishers Pvt. Ltd. A-12, 2nd Floor, Sector 2, Noida-201301 UP, India 


\section{Introduction}

Liver biopsy is indicated in both diagnosis and prognosis of diffuse liver diseases. Currently, percutaneous liver biopsy (PLB) is commonly performed because of its easy availability. However, in presence of ascites and coagulopathy (defined by international normalized ratio [INR] $>1.5$ and/or platelet count $<50000 / \mu \mathrm{L}$ ), PLB might have significant risk of fatal or nonfatal peritoneal hemorrhage that cannot be predicted by simple clinical methods. In such cases, transjugular liver biopsy (TJLB) is performed as there is less risk of peritoneal hemorrhage. Previous studies have shown that TJLB is inferior to PLB due to small size, fragmentation of specimen and yield of complete portal tracts (CPT), ${ }^{1}$ and increased risk of major complications. ${ }^{2}$ Good correlation is found between the quality of tissue sample (fragmented/small) with hepatic venous pressure gradient (HVPG). ${ }^{2}$ This retrospective study is aimed to compare the yield of tissue cores obtained through TJLB and PLB route in adults based on the total number of CPT, assess the rates of complication between these two routes, and also evaluate whether there is any significant correlation between yield of TJLB samples and HVPG.

\section{Methods}

This was a retrospective observational study. All the liver biopsies done for diffuse liver diseases between December 2017 and February 2020 at our center were included in this study. Biopsy from liver masses and repeat biopsies were excluded. The indication of liver biopsies was as follows: evaluation of suspected fatty liver disease, undiagnosed hyperbilirubinemia, raised marker of autoimmune hepatitis, to confirm a diagnosis of cirrhosis in suspected cases, and diffuse nonspecific alteration of hepatic perfusion pattern in cross-sectional imaging. A total of 123 liver biopsies were done in this period, 26 by transjugular route and 97 by percutaneous route. Hemoglobin, total lymphocyte count, differential leucocyte count, platelet, INR, serum creatinine, HBsAg, anti-HCV, and HIV I and II were routinely done before all biopsies. Two to four samples were taken for each patient in both PLB and TJLB group, according to previously reported literature. ${ }^{3-5}$ PLB was done if platelet count was above $60000 / \mathrm{mm}^{3}$, INR less than 1.5 and ascites is absent. In the presence of derangement of any of the above three parameters, TJLB was done. ${ }^{4}$ TJLB was performed under local anesthesia through the right internal jugular vein, which was accessed under ultrasound guidance and secured with a 7F sheath. Under fluoroscopy, right hepatic vein was cannulated first. Schwan-Ganz catheter was used to measure free and wedged hepatic venous pressure at the midpart of right hepatic vein using multichannel monitor. Finally, TJLB was performed with LABS-100 (Liver Access Biopsy Set, Cook, Bloomington, USA ${ }^{6}$ which comprised $19 \mathrm{G}$ biopsy needle with $60 \mathrm{~cm}$ length, fixed throw of $20 \mathrm{~mm}$, and stiff sheath through which the biopsy gun passes. Middle hepatic vein was accessed in only one case where a fragmented and small piece of tissue was obtained through the right hepatic venous approach due to the presence of significant right lobe atrophy. Middle hepatic venous access yielded satisfactory cores.

PLB was performed under local anesthesia through the right intercostal approach and ultrasound guidance with 18G Mission [Bard, Tempe, USA] disposable core biopsy instrument, followed by post biopsy gelfoam plugging for tract embolization. A $17 \mathrm{G}$ outer needle and $18 \mathrm{G}$ semiautomatic biopsy gun having $20 \mathrm{~mm}$ throw was used in all cases. After obtaining two to four cores of liver tissues, gelfoam pledgets were routinely used to embolize the biopsy tract. After biopsy, all patients were monitored overnight. Pulse, blood pressure, and oxygen saturation were monitored every 30 minutes for 4 hours with comparison between pre- and postprocedure (12 hours after biopsy) blood hemoglobin. ${ }^{7.8}$

All the biopsies were done by a single intervention radiologist, and biopsy specimens were evaluated by a single pathologist. Yield of tissue was decided based on the number of CPT. Routinely, all fields were examined under 4x magnifications and number of CPT were counted in each field. Core length is not routinely recorded at our institution. Other magnifications were also used in specific situations.

\section{Statistical Methods}

All the descriptive statistics were expressed in percentage and as mean \pm standard deviation as appropriate. Comparison of CPT between two groups was done by the two-tailed independent Mann-Whitney U test. Comparison of demographic and complication proportion between two groups were done by Chi-Squared test and Fischer exact test. Correlation of HVPG and total CPT was done by Spearman's correlation test. Significance level was kept at 0.05 . All tests were performed in Microsoft Excel version 2010.

\section{Results}

There was no statistically significant difference in mean age and gender distribution of the patients between the two groups. Patients in the TJLB group had statistically significant lower platelet count and higher INR as compared with PLB group, which is expected, based on the patient selection criteria for TJLB ( - Table $\mathbf{1}$ ). Indications of biopsy are given in

\section{- Table 2.}

Mean CPT ( - Fig. 1) in TJLB and PLB specimens are $10.9 \pm 2.7$ and $11.6 \pm 2.5$, respectively ( $p=0.566$ ). There was no statistically significant difference between the two groups. Out of $26 \mathrm{TJLB}$, none of the samples was inadequate; however, 3 out of 97 PLB samples were deemed inadequate ( - Table 3 ) for histopathological diagnosis. This difference was not statistically significant.

There was a moderate but significant negative correlation between the total number of CPT and HVPG in the TJLB group (Spearman's Rho - 0.58) ( $p=0.002)$. A major complication occurred in one case of PLB. Before the procedure, the patient did not have ascites and had a hemoglobin level of $9.2 \mathrm{~g} / \mathrm{dL}$. Twelve hours after the biopsy, she developed mild hemoperitoneum with hemoglobin drop of $1.6 \mathrm{~g} / \mathrm{dL}$. She developed hypotension $(80 / 50 \mathrm{~mm}$ of $\mathrm{Hg}$ ) and tachycardia (up to $120 /$ minute). She was managed conservatively with fluid 
Table 1 Demographic and laboratory details of patients of TJLB and PLB

\begin{tabular}{|l|l|l|l|}
\hline & TJLB $(\boldsymbol{n}=\mathbf{2 6})$ & PLB $(\boldsymbol{n}=\mathbf{9 7})$ & $\boldsymbol{p}$-Value \\
\hline Age (in years) $($ mean \pm SD) & $45.5 \pm 14.9$ & $46.5 \pm 12.9$ & 0.16 \\
\hline Female (percentage) & $17[65.3]$ & $46(47.4)$ & 0.11 \\
\hline Platelet count $\left(\mathrm{x} 10^{3} / \mathrm{mm}^{3}\right)($ Mean \pm SD) & $67.1 \pm 30.9$ & $146.3 \pm 70.8$ & 0.01 \\
\hline INR (mean \pm SD) & $1.85 \pm 0.38$ & $1.22 \pm 0.15$ & $<0.001$ \\
\hline
\end{tabular}

Abbreviations: INR, international normalized ratio; PLB, percutaneous liver biopsy; SD, standard deviation; TJLB, transjugular liver biopsy.

Table 2 Indications of liver biopsy (percentage are in parentheses)

\begin{tabular}{|l|l|l|}
\hline & TJLB $(\boldsymbol{n}=\mathbf{2 6})$ & PLB $(\boldsymbol{n}=\mathbf{9 7})$ \\
\hline Confirmation of cirrhosis & $3(11.6)$ & $35(36)$ \\
\hline $\begin{array}{l}\text { Evaluation of sus- } \\
\text { pected fatty liver } \\
\text { disease and undiagnosed } \\
\text { hyperbilirubinemia }\end{array}$ & $13(50)$ & $57(58.8)$ \\
\hline $\begin{array}{l}\text { Raised marker of autoim- } \\
\text { mune hepatitis }\end{array}$ & $9(34.6)$ & $3(3.1)$ \\
\hline $\begin{array}{l}\text { Diffuse alteration of } \\
\text { hepatic perfusion on } \\
\text { cross-sectional imaging }\end{array}$ & $1(3.8)$ & $2(2.1)$ \\
\hline
\end{tabular}

Abbreviations: PLB, percutaneous liver biopsy; TJLB, transjugular liver biopsy.

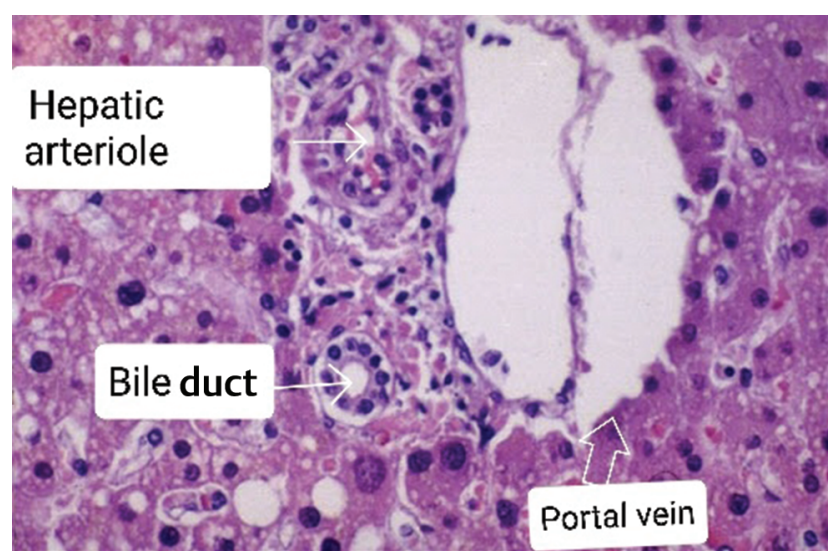

Fig. 1 Liver biopsy specimen stained with hematoxylin and eosin, seen under $40 x$ magnification showing a complete portal tract.

resuscitation and was kept under observation for the next 24 hours. No major complications occurred in the TJLB group. No death was reported in either group (- Table 4).

Minor complications, such as local site or right shoulder pain, fever $>101^{\circ} \mathrm{F}$, shivering and skin puncture site hematoma, were encountered in 4 out of 26 cases (15.3\%) of TJLB and 12 out of 97 cases (12.3\%) of PLB. There was no statistically significant difference in the rate of minor complications between the two groups $(p$ value $=0.744)(-$ Table 4$)$.

Most common biopsy findings were cirrhosis, cholestasis, steatosis and autoimmune hepatitis. Biopsy findings of TJLB and PLB are listed in - Table 5.

\section{Discussion}

Liver biopsy is used for both diagnosis and prognosis of diffuse liver diseases. PLB is routinely used and preferred because of its simplicity and safety ${ }^{9}$; however, TJLB is used in presence of coagulopathy and ascites. In this study, no statistically significant difference in major or minor complication rate was found between TJLB and PLB. In fact, there was higher proportion of both major and minor complication in PLB group despite the use of tract embolization with gelfoam plugging. Previously, major complications like subcapsular hematoma or intraperitoneal hemorrhage was reported more with TJLB. ${ }^{2}$ TJLB in this study was performed under real-time ultrasound guidance. No major complication related to Glisson capsule injury was encountered on postbiopsy venogram or in follow-up. Our finding is similar to finding of Sawyer et al who compared plugged percutaneous liver biopsy with TJLB and opined that complication rate is higher in PLB. As much as 3.5\% patients of PLB required transfusion after biopsy. No major complication was observed in the TJLB group. ${ }^{10}$ In another series of liver biopsy by Atar et $\mathrm{al},{ }^{11}$ no major complications were reported in PLB with tract embolization and TJLB. Tract embolization was initially used in coagulopathies and found to be safer. Its usefulness in PLB is also observed in previous studies. ${ }^{11}$

TJLB was historically considered inferior due to smallsized and fragmented specimens..$^{1,2,11}$ In this study, no significant difference is obtained in the number of CPT obtained through TJLB or PLB (mean CPT with standard deviation in TJLB and PLB are $10.9 \pm 2.7$ and $11.6 \pm 2.5$ ). Hong Chiang et $\mathrm{al}^{2}$ found a poor yield of specimens (51\%) in TJLB in comparison to that of PLB (95\%). One of the possible reasons can be older technique. Atar et $\mathrm{al}^{11}$ and Guido et $\mathrm{al}^{1}$ showed no statistically significant difference in TJLB and PLB, which is in accordance with our findings.

In one case of current study, fragmented sample was obtained from the right hepatic venous approach. But, on accessing middle hepatic vein, good cores were obtained. Routine use of ultrasound guidance and accessing alternate hepatic veins may improve the yield of tissue in presence of gross right lobe atrophy. ${ }^{12,13}$

Inadequate samples were obtained in 3 out of 97 patients in the PLB group. In one of them, CT showed diffusely scattered calcific nodules in the liver, and initial biopsy revealed no portal tracts. The biopsy was difficult, and needle insertion into liver parenchyma was met with rock-hard resistance. Repeat biopsy (PLB) revealed granuloma. Other patient had elevated liver transaminases and hyperbilirubinemia after liver transplant, and biopsy was done to look for acute rejection. Two passes were taken, which revealed fragmented samples with inadequate number of portal tracts. The third patient had diffuse nonspecific alteration of hepatic perfusion pattern in cross-sectional imaging, and only three portal 
Table 3 Yield of TJLB and PLB

\begin{tabular}{|l|l|l|l|}
\hline & TJLB $(\boldsymbol{n}=26)$ & PLB $(\boldsymbol{n}=\mathbf{9 7})$ & $\begin{array}{l}\text { Level of } \\
\text { significance }\end{array}$ \\
\hline CPT (mean \pm SD) & $10.9 \pm 2.7$ & $11.6 \pm 2.5$ & 0.157 \\
\hline Inadequate sampling & 0 & 3 & $>0.05$ \\
\hline Number of passes (mean) & 3.96 & 2.01 & \\
\hline
\end{tabular}

Abbreviations: CPT, complete portal tract; PLB, percutaneous liver biopsy; TJLB, transjugular liver biopsy.

Table 4 Complications of TJLB and PLB

\begin{tabular}{|l|l|l|l|}
\hline & TJLB $(\boldsymbol{n}=\mathbf{2 6})$ & PLB $(\boldsymbol{n}=\mathbf{9 7})$ & $\boldsymbol{p}$-Value \\
\hline Postbiopsy Hb drop >1 g/dL in 24 hours & 0 & 1 & - \\
\hline Hemoperitoneum & 0 & 1 & - \\
\hline Minor complication (pain, fever, local site swelling) & $4(15.3 \%)$ & $12(12.3 \%)$ & 0.744 \\
\hline
\end{tabular}

Abbreviations: PLB, percutaneous liver biopsy; TJLB, transjugular liver biopsy.

Table 5 Results of liver biopsy

\begin{tabular}{|l|l|l|}
\hline & TJLB $(\boldsymbol{n}=\mathbf{2 6})$ & PLB $(\boldsymbol{n}=\mathbf{9 7})$ \\
\hline Cholestasis & 05 & 14 \\
\hline Cirrhosis & 04 & 18 \\
\hline Chronic hepatitis & 00 & 11 \\
\hline Autoimmune hepatitis & 10 & 0 \\
\hline Steatosis & 01 & 15 \\
\hline Granuloma & 00 & 03 \\
\hline $\begin{array}{l}\text { Acute hepatitis with bridging } \\
\text { necrosis }\end{array}$ & 01 & 01 \\
\hline $\begin{array}{l}\text { Chronic hepatitis with bridg- } \\
\text { ing fibrosis }\end{array}$ & 00 & 01 \\
\hline $\begin{array}{l}\text { Infiltrative hepatocellular } \\
\text { carcinoma }\end{array}$ & 00 & 02 \\
\hline Inadequate & 00 & 03 \\
\hline Nonspecific & 02 & 07 \\
\hline Nonspecific inflammation & 01 & 00 \\
\hline Primary biliary cirrhosis & 00 & 06 \\
\hline Secondary biliary cirrhosis & 01 & 04 \\
\hline Massive necrosis & 01 & 00 \\
\hline Normal & 00 & 12 \\
\hline
\end{tabular}

Abbreviations: PLB, percutaneous liver biopsy; TJLB, transjugular liver biopsy.

tracts were obtained through PLB with no confirmed diagnosis on histopathology. It proved to be intrahepatic cholangiocarcinoma on subsequent immunohistochemistry.

In the current study, moderate but statistically significant negative correlation is found between HVPG and number of CPT. Yield of CPT is found to be significantly less in cases with raised portal pressure. Meng et $\mathrm{al}^{2}$ showed that in patients of raised HVPG, small/ fragmented samples were more commonly obtained. Higher portal pressure indicates higher fibrosis content of liver, which is postulated as the reason for poor yield.
Limitations of our study include its retrospective nature and lack of documentation of tissue core length. The study was underpowered with relatively fewer patients in the TJLB group.

\section{Conclusion}

Yield of tissue and complication rates are comparable in TJLB and PLB groups, with less complication reported in TJLB. Yield of tissue in TJLB has moderate but statistically significant negative correlation with HVPG, that is, poor yield of tissue in presence of higher portal pressure.

\section{Conflict of Interest}

None declared.

\section{References}

1 Guido M, Rugge M. Liver biopsy sampling in chronic viral hepatitis. Semin Liver Dis 2004;24(1):89-97

2 Meng HC, Lin HC, Huang CC, et al. Transjugular liver biopsy: comparison with percutaneous liver biopsy. J Gastroenterol Hepatol 1994;9(5):457-461

3 Kalambokis G, Manousou P, Vibhakorn S, et al. Transjugular liver biopsy-indications, adequacy, quality of specimens, and complications-a systematic review. J Hepatol 2007;47(2):284-294

4 Cholongitas E, Senzolo M, Standish R, et al. A systematic review of the quality of liver biopsy specimens. Am J Clin Pathol 2006;125(5):710-721

5 Miraglia R, Maruzzelli L, Minervini MI, et al. Transjugular liver biopsy in liver transplant patients using an 18-gauge automated core biopsy needle. Eur J Radiol 2011;80(3):e269-e272

6 Kis B, Pamarthi V, Fan CM, Rabkin D, Baum RA. Safety and utility of transjugular liver biopsy in hematopoietic stem cell transplant recipients. J Vasc Interv Radiol 2013;24(1):85-89

7 Govender P, Jonas MM, Alomari AI, et al. Sonography-guided percutaneous liver biopsies in children. AJR Am J Roentgenol 2013;201(3):645-650 (AJR)

8 Potter C, Hogan MJ, Kendjorsky H, Balint J, Barnard JA. Safety of percutaneous liver biopsy by interventional radiologists. J Pediatr Gastroenterol Nutr 2001;53:202-205 
9 Pathak K, Gopinath M, Salgotra KR. Transjugular liver biopsy. Med J Armed Forces India 2013;69(4):384-387

10 Sawyerr AM, McCormick PA, Tennyson GS, et al. A comparison of transjugular and plugged-percutaneous liver biopsy in patients with impaired coagulation. J Hepatol 1993;17(1):81-85

11 Atar E, Ben Ari Z, Bachar GN, et al. A comparison of transjugular and plugged-percutaneous liver biopsy in patients with contraindications to ordinary percutaneous liver biopsy and an "in-house" protocol for selecting the procedure of choice. Cardiovasc Intervent Radiol 2010;33(3):560-564

12 Jana M, Gamanagatti S. Transjugular liver biopsy: tips and tricks. Trop Gastroenterol 2012;33(3):168-172

13 Lee K, Taylor A, Bartolome B, et al. Left is alright: outcomes of transjugular liver biopsy in patients with left-lobe-only liver transplants. J Vasc Interv Radiol 2017;28(2):94-95 